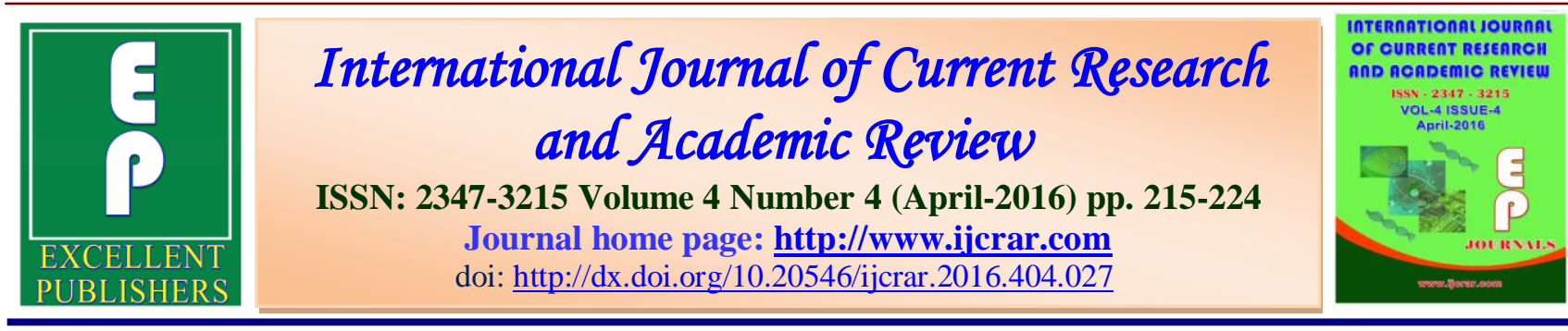

\title{
Antileishmanial activity of inosine analogs on promastigote forms of Iraqi Leishmanial species
}

\author{
Husain F. Hassan* and Iman N. Mahmood
}

Department of Biology, College of Science, University of Kirkuk, Iraq

*Corresponding author

\section{KEYWORDS}

Antileishmanial activity, inosine analogs, promastigote forms, Leishmanial species

A B S T R A C T
The growth inhibitory effects of inosine analogs, allopurinol,
thiopurinol, allopurinol riboside, formycin B, thioformycin B, 7-
deazainosine, 9-deazainosine, 8-azainosine and thio-7-deazainosine
were tested against promastigotes of Leishmania tropica, L.major and
L.donovani. All ten analogs were active against all the three species.
The efficacy and metabolism of the formycin B, allopurinol riboside,
7-deazainosine and 9-deazainosine in leishmanial species were
evaluated. These analogs affect several metabolic processes. First, is
the increased catabolism of RNA and consequent reduction of protein
synthesis. Second, inhibition of adenosine kinase, adenylosuccinate
synthetase and adenylosuccinate lyase which reduces the organisms
ability to synthesize ATP. Third, inhibition of IMP dehydrogenase and
GMP reductase ought to be sufficient to half the interconversion of
adenine nucleotides to guanine nucleotides and vice versa. The results
suggests that such inosine analogs may have promise as
antileishmanial agents.

\section{Introduction}

Pentavalent antimonials, the current drugs of choice, are widely used for treatment of leishmaniasis but frequently are not effective, often show adverse side effect and have associated toxicities at high dosages (13). Therefore, new classes of more effective and less toxic chemotherapeutic agents that are active against Leishmania are needed.
Purine metabolism in pathogenic hemoflagellate appears to offer chemotheraputically exploitable oppurtunities, not only it is different from their counter parts in human beings but also it is inhibited by pyrazolopyrimidine analogs (4-5). In addition, the enzymes of the salvage pathways are capable of accepting 
the pyrazolopyrimidine ring as a purine and metabolic inhibitor (6). This property of the hemoflagellate enzymes does not occur in humans (7) and, for this reason, pyrazolopyrimidines offer promise as potential chemotherapeutic agents for management of leishmaniasis.

The prototype of this class of compounds, allopurinol [4-hydroxypyrazolo (3,4-d) pyrimidine] is non toxic to human beings and is active against Leishmania and
Trypanosoma (8-9). Further investigation have demonstrated the therapeutic efficacy of allopurinol in cutaneous leishmaniasis and visceral leishmaniasis caused by L.tropica and L. donovani respectively.

Since allopurinol is an inosine analog (Fig. $1)$, we investigated several modifications of the inosine structure to determine which features of the molecule are important for activity against Leishmania.

Fig.1 Inosine analogs
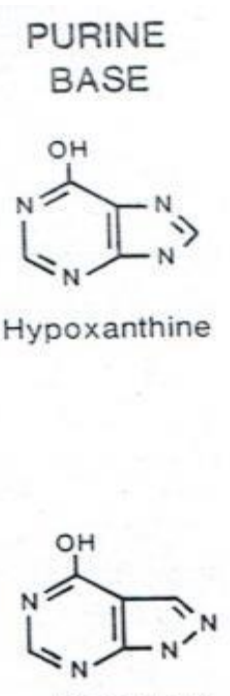

Allopurinol

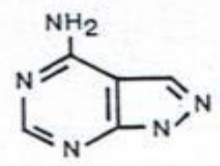

Aminopurinol

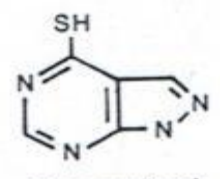

Thiopurinol

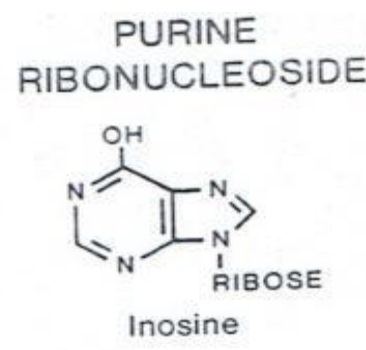

ANALOG

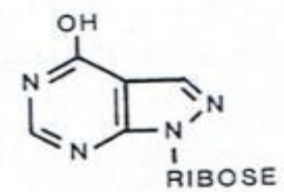

Allopurinol Ribonucleoside

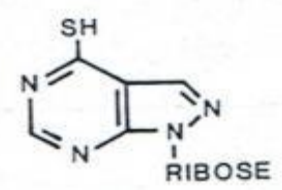

Thiopurinol Ribonucleoside
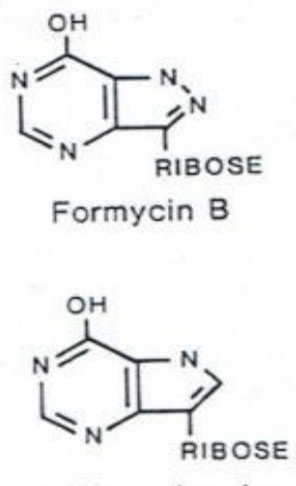

9-Deazainosine 
Int.J.Curr.Res.Aca.Rev.2016; 4(4): 215-224

Materials and Methods

\section{Growth of organisms}

Promatigotes of L.tropica, L.major,L. donovani were grown at $26 \mathrm{CO}$ in HOMEM medium supplemented with $10 \%$ (v/v) heat inactivated foetal calf serum as described previously (10). Streptomycin at $5 \mu \mathrm{g} / \mathrm{ml}$ and penicillin at $5 \mathrm{U} / \mathrm{ml}$ were added to culture media to inhibit bacterial growth.

\section{Antileishmanial effect of inosine analogs}

Cultures were initiated at 1x106 promastigotes $/ \mathrm{ml}$. The drugs were dissolved in IN $\mathrm{NaOH}$ at 50-100 times the concentrations used for experimentation and were sterilized by filtration $(0.22 \mu \mathrm{M}$ Millipore filter). One day after seeding, the promastigotes were counted using an improved Neubauer hemocytometer, and the compound to be tested was aseptically added at the desired concentration taking care to adjust the $\mathrm{pH}$ with few drops of IN $\mathrm{HCl}$ to about 7.2. Each test was made in duplicate. The number of motile parasites present in the cultures were counted daily using hemocytometer, and the counts were compared with those of controls grown in the absence of the drugs. The $50 \%$ inhibitory dose (ID50), the drug concentration that caused a 50\% reduction in growth compared with that in untreated control cultures, was determined from plots of cell growth versus drug concentration.

\section{Estimation of ribonucleic acid (RNA)}

To determine RNA content, promastigotes grown in the presence (alosence) of drug concentration causing $50 \%$ inhibition of growth were harvested by centrifugation at $3000 \mathrm{Xg}$ at $4 \mathrm{Co}$ for $10 \mathrm{~min}$ and washed twice with $0.85 \%(\mathrm{w} / \mathrm{v})$ saline. It was then precipitated by resuspension in $5 \mathrm{ml}$ of 0.2
$\mathrm{N}$ perchloric acid (PCA) at 0Co, and extracted twice at 0 Co for 30 min with 0.2 $\mathrm{N}$ PCA. Lipid was then removed by two extraction at 45 Co first with $75 \%$ (v/v) ethanol, and then with $10 \mathrm{ml}$ of ethanol/ether (1:1), Finally, nucleic acids were extracted at $70 \mathrm{Co}$ for $40 \mathrm{~min}$ with $10 \mathrm{ml}$ of $0.5 \mathrm{~N}$ PCA. The extract was then stored at $4 \mathrm{Co}$ for $48 \mathrm{hr}$, after which centrifuged at $3000 \mathrm{Xg}$ for $15 \mathrm{~min}$ with the resultant supernatant (RNA and DNA) and pellet (phospholipid) being containing deoxyribonuclease (1 $\mathrm{mg} / \mathrm{ml})$ to the standard volume $(15 \mathrm{ml})$. The amount of RNA was determined by the method of plumer (11) using an orcinol reagent with yeast RNA as standard.

\section{Determination of protein concentration}

To determine of protein content, promastigotes grown in the presence (absence) of drug concentration causing $50 \%$ inhibition of growth were harvested and washed as described above. It was then homogenized with $5 \mathrm{ml}$ of cold $5 \%$ trichloroacetic acid (TCA) to precipitate the protein. The homogenate was centrifuged at $3000 \mathrm{Xg}$ for $10 \mathrm{~min}$ with resultant supernatant and pellet being separated. The supernatant was decanted and the pellet was washed with $5 \mathrm{ml}$ of $5 \%$ TCA three times. The precipitated protein was then solubilized by IN $\mathrm{NaOH}$ for $5 \mathrm{hr}$ with continuous shaking. The supernatant which contains the protein was taken and diluted with IN $\mathrm{NaOH}$ to the standard volume $(15 \mathrm{ml})$. The protein content was estimated by the method of lowery et al (12) with bovine serum albumin as standard.

\section{Preparation of cell extract}

Mid log phase promastigotes(108cells/ml) were centrifuged at $4500 \mathrm{Xg}$ for $10 \mathrm{~min}$ at 4 CO. Pellets were washed twice in phosphate buffered saline and lysed by sonication 
involving two $15 \mathrm{sec}$ periods separated by a $30 \mathrm{sec}$ cooling period using a MSE Soniprep $150 \mathrm{sec}$ fitted with an exponential microprobe at 4 amplitude microns. Crude homogenates were centrifuged at $105000 \mathrm{Xg}$ at $4 \mathrm{Co}$ for $1 \mathrm{hr}$ and the resultant supernatant was used as source of the enzyme.

\section{Enzyme assays}

The composition of the assay mixtures and the extinction coefficients used to calculate the enzyme activities are summarised in Table 1.All enzyme activities were assayed spectrophotometrically at 26Co (13) and expressed as $\mathrm{nmol} / \mathrm{min} / \mathrm{mg}$ protein.

\section{Results and Discussion}

\section{Effects of inosine analogs on growth of Leishmania species}

As indicated in Table 2, all of these inosine analogs were inhibitory in vitro for the promastigotes of all three species of Leishmania, but their effects varied greatly from one species to another. Leishmania tropica was the most sensitive, L.major was the intermediate and L.donovani was the most resistant of the three species. The growth reduction rate took one generation (about 18 to $21 \mathrm{~h}$ ) to become fully established. When observed under the light microscope, the inosine analogs- treated promastigotes were initially elongated and they became immobile and spherical.

The inhibitory effect of the inosine analogs was leishmanistatic for all three species, because promastigotes eventually resumed their normal growth rates, reaching control cellular growth in a 4-day period, when subcultured into medium free of the drug. In general, formycin B had the lowest 50\% effective doses of the ten compounds tested in vitro. Thioformycin B was less active than formycin B. The estimated ID50 for thiopurinol was similar to that for allopurinol and the value for thiopurinol ribonucleoside was similar to that for allopurinol ribonucloside. The ID50 for 7deazainosine and 9-deazainosine were low and they compares favorably with formycin $\mathrm{B}$ in this regard. The thio dervative of 7deazainosine was relatively inactive, as was 8 -azainosine against three species of Leishmania.

A variety of purine bases (adenine, guanine, xanthine and hypoxanthine) and purine nucleoside (adenosine, guanosine, xanthosine and inosine) were used in an attempt to reverse the action of the most effective inosine analogs (formycin B, allopurinol riboside, 7-deazainosine and 9deazainosine) on three leishmanial species, at concentrations of 10 times that of the inosine analogs (Table 3 and Table 4).

Inosine was markedly effective at reversing the growth inhibition induced by inosine analogs in all three species. None of the purine bases or other purine nucleosides reversed the growth inhibition by inosine analogs in leishmanial species.

\section{Effects of inosine analogs upon RNA and protein synthesis}

To determine whether inosine analogs affect RNA metabolism, we have measured the RNA content of drug treated cells (Table 5). Formycin B, allopurinol riboside, 7deazainosine and 9-deazainosine reduced the RNA content.These effects upon RNA content are reflected in protein synthesis. Formycin B, allopurinol riboside, 7deazainosine and 9-deazainosine caused more than $80 \%$ decrease in protein content of L.tropica, L.major and L.donovani promastigotes (Table 6). 
Table.1 Spectrophotometric assay conditions

\begin{tabular}{|c|c|c|c|c|c|c|}
\hline Enzyme & EC & Substrate & $\begin{array}{c}\text { Final } \\
\text { concentration } \\
(\mathrm{mM})\end{array}$ & $\begin{array}{l}\text { Other constituent of } \\
\text { reaction mixture }\end{array}$ & product & $\mathrm{nm}\left(\mathrm{mM}^{-1} \mathrm{~cm}\right)^{\mathrm{b}}$ \\
\hline Kinase & 2.7 .1 .20 & Nucleoside & 0.5 & $\begin{array}{l}5 \mathrm{mM} \mathrm{KCl}, 1 \mathrm{mM} \mathrm{MgCl} 2,0.1 \\
\mathrm{mM} \text { PEP, } 0.1 \mathrm{mM} \mathrm{NADH}, \\
1 \mathrm{mM} \text { ATP, } 0.1 \text { UPK, } 0.1 \\
\text { ULDH }\end{array}$ & Nucleotide & $340(-18.66)$ \\
\hline Adenylosuccinate lyase & 4.3 .2 .2 & $\begin{array}{l}\text { Succino } \\
\text { AMP }\end{array}$ & 0.1 & $5 \mathrm{mM}$ EDTA & AMP & $280(-10.7)$ \\
\hline IMP dehydrogenase & 1.2 .1 .14 & IMP & 1 & $100 \mathrm{mM} \mathrm{KCL}, 0.5 \mathrm{mM}$ NAD & XMP & $340(6.22)$ \\
\hline GMP reductase & 1.6 .6 .8 & GMP & 0.1 & $\begin{array}{l}\text { 1mm EDTA, } 10 \mathrm{MM} \text { DTT, } \\
0.1 \mathrm{MM} \text { NADPH }\end{array}$ & IMP & $340(-6.22)$ \\
\hline
\end{tabular}

Abbreviations used are: IMP , inosine-5-monophosphate; GMP, guanosine-5-monophosphate; AMP, adenosine-5-monophosphate; 5-AMP, succinyladenosine-5-monophosphate; XMP, Xanthosine-5monophosphate, $\mathrm{PK}$, pyruvate kinase; $\mathrm{LDH}$, lactate dehydrogenase. .

a- The buffer used was 50mM Tris-HCl, $\mathrm{pH} 7.2$

b- Wavelength monitored (extinction coefficient).

Table.2 ID50 for various inosine analogs tested against the promastigote forms of L.tropica, L.major and L.donovani

\begin{tabular}{|l|c|c|c|}
\hline \multirow{2}{*}{ Compound } & \multicolumn{3}{|c|}{ ID $_{\mathbf{5 0}}(\boldsymbol{\mu M})$} \\
\cline { 2 - 4 } & L. tropica & L. major & L. donovani \\
\hline Allopurinol & 75 & 80 & 100 \\
\hline Thiopurinol & 70 & 88 & 96 \\
\hline Allopurinol riboside & 5 & 10 & 17 \\
\hline Thiopurinol riboside & 8 & 12 & 19 \\
\hline Formycin B & 0.06 & 0.11 & 0.8 \\
\hline Thioformycin B & 13 & 16 & 22 \\
\hline 7-Deazainosine & 0.5 & 0.9 & 1.5 \\
\hline 9-Deazainosine & 0.3 & 0.7 & 1.2 \\
\hline Thio-7-deazainosine & 35 & 36 & 41 \\
\hline 8-Azainosine & 50 & 61 & 75 \\
\hline
\end{tabular}

Note. The concentration of 9- deazainosine was $10 \mu \mathrm{g} / \mathrm{ml} /$ and that of the antagonists was 100 $\mu \mathrm{g} / \mathrm{ml}$. 
Int.J.Curr.Res.Aca.Rev.2016; 4(4): 215-224

Table.3 Reversal of the action of formycin B (FoB) on three species of Leishmania by purines

\begin{tabular}{|l|c|c|c|}
\hline \multirow{2}{*}{ Addition } & \multicolumn{3}{|c|}{ Growth of organisms compared with } \\
& \multicolumn{3}{|c|}{ control (\%) } \\
\cline { 2 - 4 } & L.tropica & L.major & L.donovani \\
\hline No FoB & 100 & 100 & 100 \\
\hline FoB & 9 & 18 & 34 \\
\hline FoB+adenine & 8 & 19 & 37 \\
\hline FoB+ guanine & 11 & 20 & 35 \\
\hline FoB+hypoxanthine & 10 & 18 & 38 \\
\hline FoB+xanthine & 11 & 16 & 36 \\
\hline FoB+adenosine & 12 & 17 & 34 \\
\hline FoB+ guanosine & 13 & 19 & 73 \\
\hline FoB +inosine & 68 & 62 & 35 \\
\hline FoB+xanthosine & 7 & 17 & 36 \\
\hline
\end{tabular}

Note. For the experiments with L.tropica, FoB was present at a concentration of $5 \mu \mathrm{g} / \mathrm{ml} /$. and the antagonists at concentrations of $50 \mu \mathrm{g} / \mathrm{m} /$.For the experiments with L.major and L.donovani, FoB was present at a concentration of $10 \mu \mathrm{g} / \mathrm{ml}$ and the antagonists at concentrations of $100 \mu \mathrm{g} / \mathrm{ml}$. The presence of the antagonists alone had no significance influence on the growth of the organisms.

Table.4 Reversal of the action of 9-deazainosine on three species of Leishmania by purines

\begin{tabular}{|l|c|c|c|}
\hline \multirow{2}{*}{ Addition } & \multicolumn{3}{|c|}{$\begin{array}{c}\text { Growth of organisms compared with } \\
\text { control (\%) }\end{array}$} \\
\cline { 2 - 4 } & L.tropica & L.major & L.donovani \\
\hline No 9-deazainosine & 100 & 100 & 100 \\
\hline 9- deazainosine & 24 & 32 & 48 \\
\hline 9- deazainosine+adenine & 20 & 34 & 46 \\
\hline 9- deazainosine+ guanine & 22 & 30 & 47 \\
\hline 9- deazainosine+hypoxanthine & 26 & 34 & 49 \\
\hline 9- deazainosine+xanthine & 24 & 29 & 44 \\
\hline 9- deazainosine+adenosine & 25 & 33 & 48 \\
\hline 9- deazainosine+ guanosine & 26 & 30 & 50 \\
\hline 9- deazainosine+inosine & 72 & 66 & 61 \\
\hline 9- deazainosine+xanthosine & 22 & 32 & 40 \\
\hline
\end{tabular}

Note: The concentration of 9- deazainosine was $10 \mu \mathrm{g} / \mathrm{ml} /$ and that of the antagonists was $100 \mu \mathrm{g} / \mathrm{ml}$. 
Int.J.Curr.Res.Aca.Rev.2016; 4(4): 215-224

Table.5 Effect of inosine analogs upon RNA synthesis

\begin{tabular}{|c|c|c|c|c|c|c|}
\hline \multirow[t]{2}{*}{ Treatment } & \multicolumn{2}{|c|}{ L. tropica } & \multicolumn{2}{|c|}{ L. major } & \multicolumn{2}{|c|}{ L. donovani } \\
\hline & $\mu \mathrm{g}$ RNA $/ 10^{8}$ & $\%$ Decrease & $\mu \mathrm{g}$ RNA $/ 10^{8}$ & $\%$ Decrease & $\mu \mathrm{g}$ RNA $/ 10^{8}$ & $\%$ Decrease \\
\hline Formycin B & $121(120-122)^{*}$ & 46 & $128(124-132)$ & 40 & $131(125-131)$ & 44 \\
\hline $\begin{array}{l}\text { Allopurinol } \\
\text { riboside }\end{array}$ & $124(119-129)$ & 45 & $135(133-137)$ & 36 & $154(150-158)$ & 34 \\
\hline 7-Deazainosine & $113(110-116)$ & 50 & $130(126-134)$ & 39 & $160(158-162)$ & 31 \\
\hline 9-Deazainosine & $142(139-145)$ & 37 & $146(145-147)$ & 31 & $157(153-161)$ & 32 \\
\hline Control*k & $224 \pm 11$ & - & $212 \pm 9$ & - & $232 \pm 14$ & - \\
\hline
\end{tabular}

*Data expressed as a mean of two experiments.

**The mean value of control $(\mu \mathrm{g} / 108$ cells $)$ is also shown.

Table.6 Effect of inosine analogs upon protein synthesis

\begin{tabular}{|c|c|c|c|c|c|c|}
\hline \multirow[t]{2}{*}{ Treatment } & \multicolumn{2}{|c|}{ L. tropica } & \multicolumn{2}{|c|}{ L. major } & \multicolumn{2}{|c|}{ L. donovani } \\
\hline & $\mu g$ Prot $/ 10^{8}$ & \% Decrease & ug Prot $/ 10^{8}$ & $\%$ Decrease & $\mu g$ Prot $/ 10^{8}$ & $\%$ Decrease \\
\hline Formycin B & $55(53-57)^{*}$ & 84 & $48(46-50)$ & 85 & $51(50-52)$ & 86 \\
\hline Allopurinol riboside & $62(60-64)$ & 82 & $58(55-61)$ & 82 & $60(56-64)$ & 83 \\
\hline 7-Deazainosine & $59(55-63)$ & 83 & $64(58-70)$ & 80 & $56(52-60)$ & 84 \\
\hline 9-Deazainosine & $64(60-68)$ & 81 & $60(57-63)$ & 81 & $62(59-65)$ & 82 \\
\hline Control*** & $340 \pm 13$ & - & $322 \pm 11$ & - & $352 \pm 15$ & - \\
\hline
\end{tabular}

*Data expressed as a mean of two experiments.

**The mean value of control $(\mu \mathrm{g} / 108$ cells) is also shown.

Table.7 Effect of inosine analogs on Leishmania tropica purine salvage enzymes

\begin{tabular}{|l|l|l|l|l|l|}
\hline \multirow{2}{*}{ Purine analogs } & \multicolumn{4}{l|}{ \% Inhibition* } & \multicolumn{4}{l|}{} \\
\cline { 2 - 6 } & Kinase & Lyase & Synthetase & Dehydrogenase & Reductase \\
\hline Formycin B & 72 & 65 & 79 & 85 & 57 \\
\hline Allopurinol riboside & 76 & 59 & 81 & 80 & 50 \\
\hline 7-Deazainosine & 69 & 61 & 73 & 89 & 55 \\
\hline 9- Deazainosine & 70 & 63 & 74 & 76 & 51 \\
\hline Control*** & $25 \pm 1$ & $3 \pm 0.5$ & $5 \pm 1$ & $51 \pm 6$ & $11 \pm 2$ \\
\hline
\end{tabular}

* Inhibitors were preincubated with the enzyme for $10 \mathrm{~min}$ at $26 \mathrm{Co}$ and the reaction was initiated by the addition of substrate.

**The mean value of control represent the specific activity expressed as $\mathrm{nmol} / \mathrm{min} / \mathrm{mg}$ protein

Table.8 Effect of inosine analogs on Leishmania major purine salvage enzymes

\begin{tabular}{|l|c|c|c|c|c|}
\hline \multirow{2}{*}{ Purine analogs } & \multicolumn{5}{|c|}{ \% Inhibition* } \\
\cline { 2 - 6 } & Kinase & Lyase & Synthetase & Dehydrogenase & Reductase \\
\hline Formycin B & 69 & 57 & 82 & 76 & 52 \\
\hline Allopurinol riboside & 67 & 59 & 78 & 75 & 53 \\
\hline 7-Deazainosine & 70 & 62 & 70 & 69 & 54 \\
\hline 9- Deazainosine & 72 & 65 & 73 & 72 & $16 \pm 3$ \\
\hline Control** & $19 \pm 1$ & $2 \pm 0.1$ & $6 \pm 0.5$ & $63 \pm 3$ & \\
\hline
\end{tabular}

* Inhibitors were preincubated with the enzyme for $10 \mathrm{~min}$ at $26 \mathrm{Co}$ and the reaction was initiated by the addition of substrate.

**The mean value of control represent the specific activity expressed as $\mathrm{nmol} / \mathrm{min} / \mathrm{mg}$ protein. 
Int.J.Curr.Res.Aca.Rev.2016; 4(4): 215-224

Table.9 Effect of inosine analogs on Leishmania donovani purine salvage enzymes

\begin{tabular}{|l|c|c|c|c|c|}
\hline \multirow{2}{*}{ Purine analogs } & \multicolumn{5}{c|}{ \% Inhibition* } \\
\cline { 2 - 6 } & Kinase & Lyase & Synthetase & Dehydrogenase & Reductase \\
\hline Formycin B & 73 & 61 & 74 & 80 & 58 \\
\hline Allopurinol riboside & 61 & 66 & 70 & 83 & 56 \\
\hline 7-Deazainosine & 68 & 59 & 71 & 77 & 57 \\
\hline 9- Deazainosine & 67 & 60 & 76 & 74 & 53 \\
\hline Control*** & $31 \pm 5$ & $6 \pm 1$ & $9 \pm 2$ & $59 \pm 4$ & $20 \pm 2$ \\
\hline
\end{tabular}

* Inhibitors were preincubated with the enzyme for $10 \mathrm{~min}$ at $26 \mathrm{Co}$ and the reaction was initiated by the addition of substrate.

**The mean value of control represent the specific activity expressed as $\mathrm{nmol} / \mathrm{min} / \mathrm{mg}$ protein.

To determine wether the reduced RNA content of inosine analogs - treated cells was due to inhibition of purine salvage enzymes, cells were extracted and assayed for enzymatic activites. A significant inhibition of nucleoside kinase, adenylosuccinate synthestase, adenylosuccinate lyase and IMP dehydrogenase was seen in promastigotes of all three species of Leishmania exposed to inosine analogs (Table 7-9). These results indicate that the increased catabolism of RNA caused by the inosine analogs modulates the utilisation of exogenous purine.

The observation reported here suggest that inosine analogs provide good models for the design of agents with antileishmanial activity. Of the ten different analogs tested, four compounds (formycin B, 9deazainosine, 7-deazainosine and allopurinol riboside) were the most promising, as they strongly inhibited multiplication of culture forms of three species of the Leishmania. In addition, it was found that L.donovani was less sensitive to inosine analogs than L.tropica and L.major. The reason for this differences in unclear, but it may reflect quantitative differences in either transport or enzymatic activites between the three organisms. Similar difference among various Trypanosoma species with respect to inosine analogs sensitivity have also been found (7).
The effects of allopurinol, thiopurinol and their ribonucleosides on the growth of these leishmaniae were somewhat different. Allopurinol riboside and thiopurinol riboside were more active than the bases allopurinol and thiopurinol, against L.tropica, L.major and L.donovani. The inhibition by allopurinol riboside and thiopurinol riboside were reversed by inosine, whereas inosine had no effect on that caused by allopurinol and thiopurinol. The similar antileishmanial activity of allopurinol to thiopurinol and of allopurinol riboside to thiopurinol riboside in this model confirms the data from infected tumer macrophages(14-15). However, the apparent mechanism of allopurinol riboside and thiopurinol riboside is that they are metabolized to ribotide by the parasites nucleoside kinase, which then incorporate in to RNA (16). It has been reported here, that allopurinol riboside and thiopurinol riboside also inhibit nucleoside kinase activity, and this could be due to their competing as substrates for the parasite enzymes(6). These observations provide one possible explanation as to how inosine antagonise the antileishmanial activities of allopurinol riboside and thiopurinol riboside.

The vastly increased antileishmanial activity of formycin B compared with allopurinol riboside indicates that leishmanial promastigotes clearly distinguish these two close analogs of inosine. Promastigotes in 
this model do not distinguish between allopurinol riboside and thiopurinol riboside, but the organisms do distinguish formycin B from thioformycin $\mathrm{B}$ and 7-deazainosine from thio-7-deazainosine. However, the differential activity of these inosine analogs compared with their thio derivatives might not be clinically expoitable.

7-deazainosine and 9-deazainosine are of considerable interest not only because they supports the general theme of inosine analogs as potential chemotherapeutic agents for several protozoan diseases but also because they have a carbon-carbon bond between the heterocyclic ring and the ribose. This bond is not broken in mammalian cells and these compounds should resist metabolic degradation in humans (7).

The data presented here suggest that inosine analogs (Formycin B, 9-deazainosine, 7deazainosine and allopurinol riboside) inhibit the growth of leishmanial promastigotes by inhibition of enzymes which carry out interconversion of purine nucleotide and by the mechanisms related to decrease in the net amount of RNA per cell and its loss of function, as measured by protein synthesis. Promastigotes are surrounded by high concentrations of host cell ATP and GTP. Presumably, these nucleotides are utilized by the parasite following cleavage to their respective ribonucleosides or bases and under these conditions purine interconversion enzymes would be necessary. The decreased in amount of RNA and the inhibition of protein synthesis which are related to the inosine analogs concentration suggest that there are one or more other mechanisms of inhibition of cell growth active at the level of RNA function. An inosine analog which can be phosphorylated and aminated by a protozoan system, but not by a mammalian system would be an excellent candidate agent for antiprotozoan chemotherapy.

\section{References}

1.Croft, S. L. and Yardly.(2002). Chemotherapy of leishmaniasis. Current Pharmaceutical Design. 8,PP. 319-342.

2.Croft, S.L. and Olliaro, P.(2011). Leishmaniasis chemotherapychallenges and opportunities.Clin Microbiol Infect. 17,PP. 1478-1483.

3.Jardim,I.Z., Thiemann, O.H. and Anibal, F.F.(2014). Leishmaniasis and Chagas Disease Chemotherapy: a Critical Review. J. Braz. Chem. Soc. 25, 18101823.

4.Hassan, $\mathrm{H}$ and Coombs, G(1988). Purine and pyrimidine metabolism in parasitic protozoa. FEMS Microbiol., 54,47-54.

5.Hasan, A., Satyanarayana, M., Mishra, A., Bhakuni, D. S., Pratap, Ram, Dube, A. and Guru, P. Y.(2006) 'Acyclic Pyrazolo[3,4-d]Pyrimidine Nucleoside as Potential Leishmaniostatic Agent', Nucleosides, Nucleotides and Nucleic Acids, 25, 55 - 60.

6.Hassan, H.(2002). Effect of some purine analogs on growth and metabolism of leishmania major promastigotes.2nd Int. Con. Biol.Sci.,Egypt,P.51..

7.Marr, J.(1991). Purine analogs as chemotherapeutic agent in leishmaniasis and American trypanosomiasis. J. Lab.Clin. Med., 118,111-119..

8.Yasur-Landau D, Jaffe CL, David L, Baneth G.(2016). Allopurinol Resistance in Leishmania infantum from Dogs with Disease Relapse. PLoS. Negl Trop Dis.10,1-13.

9.Chawla, B. and Madhubala, R.(2010). Drug targets in Leishmania. J Parasit Dis.34, 1-13. 
10.Hassan, H. and Coombs, G.(1986). Comparative study of the purine and pyrimidine metabolising enzymes of a range of trypanosomatids. Comp. Biochem. physiology.,84,217-223.

11.Plumer, D. (1978). An Introduction to Practical Biochemistry. 2nd. Ed., McGrawhill Book Com., Ltd., England, uk.

12.Lowery, O., Rosenbrough, N., Farr, A. and Randall, R.(1951). Protein measurement with folin phenol reagent. J.Biol.Chem., 193,265-275..

13.Hassan, H. and Coombs, G. (1985). Leishmania mexicana: Purine metabolizing enzymes of amastigotes and promastigotes. Exp. Parasitol., 59,139-150..

14.Mur, J.,Berens,R., Nelson, D.,Krenitsky, T., Spector, T., Lafon, S. and Elion, G. (1982). Antileishmanial action of 4thiopyrazolo $(3,4-d)$ pyrimidine and its ribonucleosides. Biochem. Pharmacol., 31, 143-148.
15.Marr, J. (1983). Pyrazolopyrimidine metabolism in leishmania and trypanosomes. J. Cell. Biochem., 22,187-196.

16.Nelson, D., Bugge, C., Elion G., Berens, R. and Marr, J. (1979). Allopurinol ribonucleoside as an antileishmanial agent. Bioclogical effects, metabolism and enzymatic phosphorylation. J. Biol. Chem., 254,11544-115449..

17.Spector, T. and Jones, T. (1982). Guanosine-5-monophosphate reductase from Leishmania donovani: Apossible chemotherapeutic target. Biochem. Pharmacol., 31, 3891-3897.. 18.Spector, T., Jones, T., Lafon, S., Nelson, D., Berens, R. and Marr, J.(1984). Monophosphates of formycin B and allopurinol riboside: Interaction with leishmanial and mammalian succinoAMP synthetase and GMP reductase. Biochem. Pharmacol., 33, 1611-1617.

\section{How to cite this article:}

Husain F. Hassan and Iman N. Mahmood. 2016. Antileishmanial activity of inosine analogs on promastigote forms of Iraqi Leishmanial species. Int.J.Curr.Res.Aca.Rev. 4(4): 215-224. doi: http://dx.doi.org/10.20546/ijcrar.2016.404.027 